\section{Targeting tankyrase}

\section{By Lev Osherovich, Senior Writer}

Researchers from the University of Oslo and Roche's Genentech Inc. unit have developed several selective inhibitors of tankyrases TNKS and TNKS2, two druggable targets in the otherwise hard-to-hit WNT pathway. ${ }^{1,2}$ The university team's lead compound has been licensed to Odin Therapeutics A/S for colorectal cancer.

Excessive signaling by the wingless-type MMTV integration site (WNT) pathway drives cell regeneration in a range of solid tumors, but companies have struggled to find therapeutically tractable targets. The tankyrases-a pair of enzymes that normally work by modifying a range of cellular proteins with ADP-ribose-are the best-validated downstream target for inhibiting WNT signaling, according to Genentech senior scientist in the Department of Cancer Targets Mike Costa, whose team has a project to identify new WNT pathway targets.

In 2009, researchers at Novartis AG's Novartis Institutes for BioMedical Research established the connection between tankyrases and cancer by identifying TNKS (tankyrase TRF1-interacting ankyrinrelated ADP-ribose polymerase) and TNKS2 as the targets of antitumor compound XAV939. ${ }^{3}$ Since then, XAV939's poor selectivity has kept it from advancing into the clinic.

XAV939 hits not only the two tankyrase enzymes but also other members of the poly(ADP-ribose) polymerase (PARP) family.

Now, a team led by Stefan Krauss, professor of microbiology at the University of Oslo and director of stem cell signaling at Oslo University Hospital, has designed a new class of tankyrase inhibitor that hits a binding site on the enzyme that is different from that found in other PARP family members.

"We have a highly specific tankyrase inhibitor," said Krauss. "If you just want to inhibit tankyrase, the way forward seems to be to target the adenosine binding site," which is distinctive in tankyrases.

Krauss teamed up with Costa to obtain in vivo, proof-of-concept data for Krauss' compound as well as for an unrelated tankyrase inhibitor, G244-LM, discovered independently at Genentech.

"A number of groups have published compounds that inhibit signaling in cell culture, but ours are the first compounds to have enough stability to inhibit tumor growth in vivo," said Costa.

\section{SAR land}

Krauss' team ran an SAR study to find potent derivatives of an earlier 1,2,4-triazole compound made by his team that blocked the adenosine binding pocket of TNKS and TNKS2. The best compound to emerge from the SAR study, named G007-LK, had in vitro $\mathrm{IC}_{50}$ values of $46 \mathrm{nM}$ for TNKS and $25 \mathrm{nM}$ for TNKS2. The molecule also inhibited tankyrase activity at a comparable concentration in cell culture.
Unlike XAV939, G007-LK did not appreciably inhibit PARP-1 in vitro. PARP-1 lacks an adenosine binding pocket.

G007-LK had good oral availability in mice and about a three-hour half-life when administered intravenously, suggesting the compound was sufficiently stable to be a drug candidate.

Krauss then collaborated with Costa to evaluate G007-LK in mouse xenograft tumors with mutations in adenomatous polyposis coli (APC), a tumor suppressor whose absence drives the growth of many colorectal cancers due to excessive WNT signaling.

In cell culture, G007-LK and G244-LM both decreased WNT signaling compared with vehicle. Both compounds led to higher levels of axin proteins, which counteract the tumor-driving effect of $\beta$-catenin (CTNNB1), a transcription factor that is the downstream effector of the WNT signaling pathway (see Figure 1, "Targeting tankyrase to treat tumors").

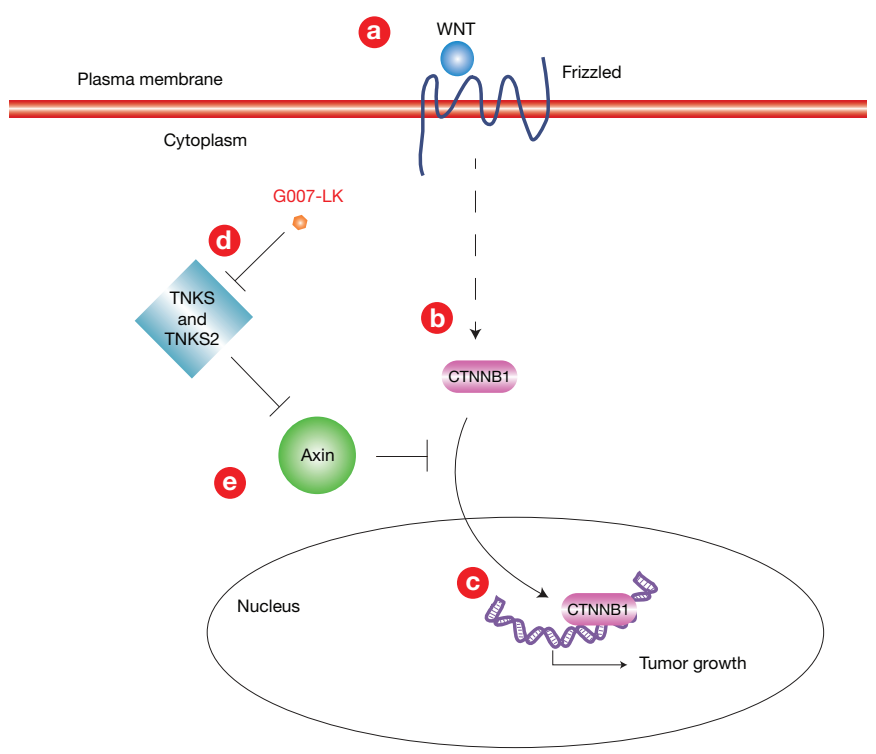

Figure 1. Targeting tankyrase to treat tumors. Lau et al. and Voronkov et al. have shown that small molecule inhibitors of tankyrase TRF1-interacting ankyrin-related ADP-ribose polymerase (TNKS) and TNKS2 can slow the growth of colorectal cancer xenografts in mice.

In colorectal cancer, wingless-type MMTV integration site (WNT) signaling through various frizzled receptors [a] leads to stabilization of $\beta$-catenin (CTNNB1), which enters the nucleus [b] and turns on genes that drive tumor proliferation [c].

Lau et al. and Voronkov et al. found that G007-LK [d], a small molecule inhibitor of TNKS and TNKS2 that is licensed to Roche's Genentech Inc. unit, prevents this series of events. TNKS and TNKS2 cause the degradation of axin proteins, which counteract the accumulation of CTNNB1. G007-LK treatment leads to stabilization of axins and blocks CTNNB1 activity [e], thus inhibiting tumor growth. 


\section{ANALYSIS}

G244-LM is chemically related to XAV939 and is thought to block the same nicotinamide binding pocket of tankyrases as the latter.

In mouse xenograft models for colorectal cancer driven by APC mutations, G007-LK decreased tumor growth compared with vehicle. Not all APC mutant cell lines were sensitive to the tankyrase inhibitors. In vivo data for G244-LM were

\section{"A number of groups have published compounds that inhibit signaling in cell culture, but ours are the first compounds to have enough stability to inhibit tumor growth in vivo." \\ -Mike Costa, Genentech Inc.} not reported.

SAR and pharmacokinetic results were reported in the Journal of Medicinal Chemistry. Mouse efficacy data were reported in Cancer Research.

\section{Twilight of the tumors}

The biggest challenge to further development of G007-LK is its tolerability profile. In healthy mice, the compound blocked the regeneration of intestinal crypt cells, a normal process that depends on WNT signaling.

"We have to establish whether this intestinal toxicity is reversible," said Costa. "We think this is an on-target, mechanism-based toxicity. Tankyrase inhibitors at high doses are able to block crypt cell proliferation, an expected effect of inhibiting this pathway."

Costa said tankyrase inhibitors do not directly kill tumors but instead appear to block the self-renewal of undifferentiated cells with stem cell-like properties that are thought to be the source of colorectal tumors. Blocking tankyrases appears to drive those cells into a more differentiated state, thus reducing the supply of cancer stem cells.

"We know that treating xenograft tumors with tankyrase inhibitors induces differentiation biomarkers, so it's possible that this is inhibiting self-renewal," said Costa.

Costa's team is now optimizing the treatment regimen to reduce the compounds' toxicity. One potential approach is to use "combination therapies that might allow us to lower the dose," he said.

Krauss said tankyrase inhibitors would only work against tumors with high WNT signaling activity and that clinical biomarker studies are needed to figure out which patients will most likely respond to tankyrase inhibitors.

"The major challenge will be patient inclusion or exclusion," said
Krauss. "These are not compounds that would work on all colorectal cancer patients. It's very important to find out the context in which tankyrase inhibitors could work as a single-agent treatment and circumstances where they may work as part of a two-compound regimen."

Both Krauss and Costa said high WNT activity is found in other solid tumors, including some cases of non-small cell lung cancer (NSCLC).

Meanwhile, Krauss has filed for patents covering G007-LK and related compounds through the technology transfer office of the University of Oslo. Those patents have been licensed to Odin. Krauss said the biotech is raising VC funding for further development of its compounds.

Genentech spokesperson Nadine Pinell said the company has not licensed G007-LK and has not yet selected a preclinical candidate from among its own tankyrase inhibitors.

Osherovich, L. SciBX 6(15); doi:10.1038/scibx.2013.353

Published online April 18, 2013

\section{REFERENCES}

1. Voronkov, A. et al. J. Med. Chem.; published online March 11, 2013; doi:10.1021/jm4000566

Contact: Stefan Krauss, Oslo University Hospital, Oslo, Norway

e-mail: stefan.krauss@rr-research.no

Contact: Jens P. Morth, same affiliation as above

e-mail: j.p.morth@ncmm.uio.no

Contact: Jo Waaler, same affiliation as above e-mail: jo.waaler@rr-research.no

2. Lau, T. et al. Cancer Res.; published online March 28, 2013; doi:10.1158/0008-5472.CAN-12-4562

Contact: Mike Costa, Genentech Research \& Early Development, South San Francisco, Calif.

e-mail: mcosta@gene.com

3. Huang, S.-M.A. et al. Nature 461, 614-620 (2009)

COMPANIES AND INSTITUTIONS MENTIONED

Genentech Inc., South San Francisco, Calif.

Novartis AG (NYSE:NVS; SIX:NOVN), Basel, Switzerland

Novartis Institutes for BioMedical Research, Basel, Switzerland

Odin Therapeutics A/S, Oslo, Norway

Oslo University Hospital, Oslo, Norway

Roche (SIX:ROG; OTCQX:RHHBY), Basel, Switzerland

University of Oslo, Oslo, Norway 